\title{
artigo
}

Gomes da Silva, E.; Maciel, J.R.; Pontes, A.C.; Delfino, V.D.F.R.; Gomes da Silva, F.; Pinheiro Junior, J.A.P.;

Adaptações de uma unidade de pronto atendimento frente a pandemia do COVID-19: relato de experiência

\section{Adaptações de uma unidade de pronto atendimento frente a pandemia do COVID-19: relato de experiência}

\author{
Adaptations of an emergency care unit in front of the COVID-19 pandemic: experience report \\ Adaptaciones de una unidad de atencion de emergencias frente a lá pandemia covid-19: informe de experiencia
}

\begin{abstract}
RESUMO
Objetivo: relatar as principais experiências vivenciadas por profissionais que atuaram na linha de frente em uma UPA no município de Parnamirim-RN. Método: Trata-se de um relato de experiência sobre as adaptações sofridas em uma unidade de pronto atendimento durante a pandemia do COVID-19. 0 período em questão do estudo repercute o cotidiano da instituição entre março e dezembro de 2020. O relato foi elaborado e baseado de acordo com vivências dos profissionais que atuaram na linha de frente dentro da instituição. Resultados: Diversas adaptações foram necessárias durante a pandemia, a estrutura física da instituição, novos protocolos precisaram ser seguidos, educação em saúde para o uso de equipamentos de proteção individual e adaptações para a nova realidade. Conclusão: Ficou evidente que houve modificações significativas durante a pandemia, seja na abordagem de trabalho individual e em equipe, protocolos operacionais, mudanças estruturais e de fluxo de atendimento que refletiram o cenário estabelecido.
\end{abstract}

DESCRITORES: Urgência; Emergência; Pandemia; Infecções por Coronavírus.

\section{ABSTRACT}

Objective: to report the main experiences lived by professionals who worked in the front line in a UPA in the municipality of Parnamirim-RN. Method: This is an experience report about the adaptations suffered in an emergency care unit during the COVID-19 pandemic. The period in question of the study reflects the daily life of the institution between March and December 2020. The report was prepared and based on the experiences of the professionals who worked on the front lines within the institution. Results: Several adaptations were necessary during the pandemic, the physical structure of the institution, new protocols needed to be followed, health education for the use of personal protective equipment and adaptations for the new reality. Conclusion: It was evident that there were significant changes during the pandemic, whether in the approach of individual and team work, operational protocols, structural changes and the flow of care that reflected the established scenario.

DESCRIPTORS: Urgency; Emergency; Pandemic; Coronavirus Infections.

\section{RESUMEN}

Objetivo: reportar las principales experiencias vividas por los profesionales que trabajaron en primera línea en una UPA en el municipio de Parnamirim-RN. Método: Se trata de un relato de experiencia sobre las adaptaciones sufridas en una unidad de atención de emergencia durante la pandemia de COVID-19. El período en cuestión del estudio refleja la vida cotidiana de la institución entre marzo y diciembre de 2020. El informe fue elaborado y basado en las experiencias de los profesionales que trabajaron en primera línea dentro de la institución. Resultados: Fueron necesarias varias adaptaciones durante la pandemia, la estructura física de la institución, nuevos protocolos a seguir, educación en salud para el uso de equipos de protección personal y adaptaciones a la nueva realidad. Conclusión: Se evidenció que hubo cambios significativos durante la pandemia, ya sea en el enfoque del trabajo individual y en equipo, protocolos operativos, cambios estructurales y el flujo de atención que reflejó el escenario establecido.

DESCRIPTORES: Urgencia; Emergencia; Pandemia; Infecciones por coronavirus.

RECEBIDO EM: 29/01/2021 APROVADO EM: 15/02/2021

\section{Edineide Gomes da Silva}

Enfermeira, graduada pela Faculdade de Enfermagem/Universidade do Estado do Rio Grande do Norte (UERN).

ORCID: 0000-0002-1573-3341 


\section{Julyana Rodrigues Maciel}

Enfermeira, graduada pela Faculdade de Enfermagem/Universidade do Estado do Rio Grande do Norte (UERN).

ORCID: 0000-0002-3060-234X

\section{Alan Campelo Pontes}

Enfermeiro, graduado pela Faculdade Nova Esperança (FACENE).

ORCID: 0000-0002-5110-8893

\section{Victória D'awylla Ferreira Rocha Delfino}

Enfermeira, graduada pela Faculdade de Enfermagem/Universidade do Estado do Rio Grande do Norte (UERN).

ORCID: 0000-0003-4517-2634

\section{Fernanda Gomes da Silva}

Enfermeira, graduada pela Faculdade de Enfermagem/Universidade do Estado do Rio Grande do Norte (UERN). ORCID: 0000-0001-9919-8544

\section{José Adaísio Pinheiro Junior}

Acadêmico de Medicina, pela Universidade do Estado do Rio Grande do Norte (UERN).

ORCID: 0000-0002-9473-3883

\section{INTRODUÇÃO}

A população mundial vem encarando desde janeiro de 2020 uma pandemia ocasionada pela emergência do novo coronavírus. O SARS-Cov-2, causador da COVID-19. Descoberto em Wuhan na China em 01 de dezembro de 2019, devido sua alta taxa de transmissibilidade, disseminou-se rapidamente por todo o mundo, provocando uma grave crise sanitária global ${ }^{1}$.

O SARS-Cov-2 é um patógeno que afeta o sistema respiratório desencadeando manifestações clínicas que variam desde infecções respiratórias sem sintomas, até um quadro grave. Segundo as estimativas da Organização Mundial de Saúde (OMS), 80\% dos pacientes acometidos pela COVID-19 apresentam sintomas leves, $15 \%$ requerem internação hospitalar apresentando dificuldade respiratória e $5 \%$ dos pacientes evoluem para forma grave da doença necessitando de suporte ventilatório ${ }^{2,3}$.

A transmissão do vírus acontece, principalmente, por meio de gotículas contaminadas de secreção da orofaringe, pelo espirro e saliva, de uma pessoa infectada para outra não contaminada. $\mathrm{O}$ período de incubação é em média 5 dias, podendo variar de 1 a 14 dias. O tempo prolongado de incubação contribui para o alto índice de propagação, tendo em vista que pesso- as assintomáticas, pré-sintomáticas e com sintomas leves transmitem a doença ${ }^{4}$.

No Brasil, desde o primeiro caso confirmado em 26 de fevereiro de 2020 até 16 de janeiro de 2021, o total de casos confirmados da doença já somam 8.455.059, destas 209.296 pessoas vieram a óbito. Um total de 2.026 .763 e 49.672 casos, respectivamente, aconteceram no Nordeste. No Rio Grande do Norte (RN), notificou-se 129.549 casos confirmados e 3.173 óbitos. Em Parnamirim foram notificados 12.754 casos dessas 228 pessoas já foram a óbito ${ }^{5}$.

A emergência da pandemia pela $\mathrm{CO}$ VID-19 trouxe inúmeros desafios para seu enfrentamento, com grande impacto negativo na economia, assistência médica e saúde mental, levando em conta a escassez do conhecimento científico acerca do coronavírus, sua alta capacidade de transmissão e elevado potencial de mortalidade em público-alvo, causando incertezas sobre quais medidas são eficazes para seu combate. No Brasil o cenário torna- se ainda mais complexo, considerando as condições precárias de habitação e saneamento, em situação de aglomeração e com acesso ao sistema de água deficiente ${ }^{6}$.

O cenário de pandemia exigiu adaptação e reorganização assistencial e estrutural nas instituiçôes e serviços de todos os níveis de complexidade, como a ampliação de leitos hospitalares, contratação de profissionais capacitados, fornecimento de equi- pamentos de proteção individual (EPI) e insumos suficientes, criação de alas de isolamento, organização de fluxos assistenciais, elaboração de protocolos, remanejamento de profissionais, desenvolvimento de capacitaçôes, dentre outras ações necessárias para atender a população ${ }^{7,8}$.

Tendo em vista o contexto de adaptações dos serviços e os inúmeros desafios enfrentados para o combate a COVID-19, surgiu o seguinte questionamento: quais adaptações foram necessárias durante a pandemia numa instituição de urgência e emergência? Assim o presente trabalho objetiva relatar essas adaptações através de experiências vivenciadas por profissionais que atuaram na linha de frente em uma Unidade de Pronto Atendimento (UPA) no município de Parnamirim-RN.

\section{METODOLOGIA}

Trata-se de um estudo de caráter descritivo do tipo relato de experiência que aborda as vivencias de profissionais de uma Unidade de Pronto Atendimento sobre a reorganização sua percepção acerca das modificações estruturais, organizacionais e de gerência ocorridas na mesma, no início do período de pandemia da COVID-19 e suas repercussões para o funcionamento e fluxo de trabalho.

A unidade Maria Nazaré referenciada no presente trabalho foi escolhida por 
ser porta de entrada para tratamento de pacientes acometidos pela COVID-19 durante a pandemia, situada na região Nordeste, estado do Rio Grande do Norte, especificamente no município de Parnamirim e classificada como UPA nível 3, com atendimentos de média complexidade em urgência e emergência.

A instituição oferece atendimentos divididos de acordo com a cor da classificação de risco: azul, verde, amarelo ou vermelho. Atribuídas com base na gravidade de risco de acordo com o Protocolo de Manchester. Dessa forma a unidade oferece atendimentos a pacientes exclusivamente pelo Sistema Único de Saúde (SUS).

O período relatado no estudo e as demandas exemplificadas compreendem o período de março e dezembro de 2020. Os dados relatados foram baseados de acordo com as vivências dos profissionais que atuaram na linha de frente no enfrentamento ao quadro da doença do COVID-19 dentro da instituição.

\section{RESULTADOS}

\section{Adaptações do serviço}

As necessidades de inúmeras adaptações ao serviço foram necessárias desde o início da pandemia. Deste modo, houve a realidade de um quantitativo de profissionais precisarem se afastar, devido se enquadrarem como grupo de risco para o COVID-19, outros em determinado momento se contaminaram com o vírus e também foram afastados. Constantemente, ocorreu um alto fluxo de profissionais dando entrada na instituição, devido a necessidade de uma maior demanda frente ao estado de calamidade pública enfrentado.

A estrutura física da instituição precisou de modificações para dar melhor suporte tanto aos pacientes como também aos funcionários. Além disso, foram criadas estratégias de comunicação, como alertas visuais (cartazes, placas e etc.) nos setores da unidade mediante locais específicos.

Dispensadores de álcool foram redistribuídos por todos os locais de atendimento. Espaços de isolamentos precisaram ser criados, como por exemplo os consultórios se transformaram em salas de isola- mento adaptadas para receber os pacientes suspeitos ou confirmados do COVID-19. Assim, foi necessário aderir um setor específico para isolamento, afim de dar suporte exclusivo aos casos da doença.

Equipes precisaram ser escaladas especialmente para atender esses leitos de isolamento, com a finalidade de não disseminar a contaminação do vírus dentro da instituição. Surgiu novos protocolos que começaram a ser seguidos. A triagem dos pacientes ganhou uma nova abordagem. Nos atendimentos o foco principal passou a ser a orientação as pessoas sobre a doença, a fim de evitar mais contaminação.

A Educação em Saúde foi primordial como forma de prevenção da doença, durante toda abordagem do serviço. Cuidados básicos de higiene passaram a ser a temática central nos atendimentos, enaltecendo a importância de orientar os pacientes a evitar exposição aos riscos.

Alguns procedimentos e tratamentos começaram a ser suspensos devido ao risco de exposição aos aerossóis que aumentam a contaminação da doença. Um deles foi o não uso da nebulização, assim como o procedimento de aspiração de pacientes em sistemas abertos, também foi suspenso ambuzar pacientes suspeitos ou confirmados de COVID-19.

A tramitação de todo e qualquer profissional com cuidados diretos a esses pacientes condicionou a mudanças na sua segurança, que se refletiram no cuidado direto ao risco da exposição da doença. EPIs foram modificados: máscaras cirúrgicas nos atendimentos de rotina passaram a ser substituídas pelo uso do modelo N95, assim como a obrigatoriedade na utilização de capotes, face-shields, gorros, e aventais.

\section{Desafios vivenciados}

Devido ao alto risco de contaminação do COVID-19, acompanhantes passaram a não ser permitidos dentro da instituição, a fim de evitar o aumento da contaminação pelo vírus. A própria equipe de tramitação dentro dos setores passou a ser restrita. Por ter alta taxa de transmissibilidade o COVID-19 atingiu uma grande quantidade de infectados rapidamente.
A inexistência de tratamento específico para a doença, vacina, medicamentos, testes insuficientes para a população, e tantas incertezas sobre a doença colocou os profissionais da instituição em exaustiva carga de trabalho. EPIs desconfortáveis que machucavam a pele, equipes desfalcadas, fluxo de novos profissionais que chegavam e precisavam pegar a rotina do serviço, muitos inseridos sem experiência alguma na urgência e emergência.

$\mathrm{O}$ medo da contaminação. A falta de informações mais específicas sobre a doença. O temor de não poder abraçar os entes queridos, o estresse do trabalho, a falta de equipamentos essenciais, informações fiéis sobre a doença, medicamentos, suporte respiratório suficientes para tanta demanda dos pacientes hospitalizados. A ameaça do colapso dos serviços de saúde se fez presente a todo momento. A doença passou rapidamente de transmissão comunitária para seu crescimento exponencial.

Foram dias difíceis, muita dor, morte, perdas irreparáveis tanto de pacientes, como de pessoas da própria equipe. Contudo o serviço passou a ser mais adaptado com o passar dos meses. Exames mais específicos começaram a ser disponibilizados, toda uma logística de referência passou a ser existente. Os profissionais passaram a ser mais qualificados na linha de frente. Novos protocolos foram criados, e apesar de muitas dificuldades e incertezas a esperança de dias melhores começou a fazer parte da rotina de trabalho dentro da instituição.

\section{DISCUSSÃO}

As diversas adaptações geradas no serviço de saúde durante a pandemia repercutiram grandes desafios na rotina de trabalho da instituição. A rotatividade de profissionais foi um grande desafio enfrentado. A OMS recomendou remanejar ou afastar os trabalhadores pertencentes ao grupo de risco desde o início da pandemia 5 .

Muitos foram afastados também devido a contaminação com o vírus. Vários precisaram ser recrutados para dar suporte à nova demanda. Profissionais foram inseridos no serviço sem nenhuma experiên- 
cia, todos sem saber ao certo o que estava realmente enfrentando. Equipes desfalcadas, e muita demanda de pacientes contaminados sobrecarrega o serviço.

As estratégias de comunicação se mostraram importantes aliados para a conscientização da população sobre os riscos da doença. Em relação às adaptações no espaço da instituição, segundo as recomendações da Organização Pan-Americana da Saúde - OPAS, o aumento do espaço físico para acomodar esses pacientes foi de suma importância para melhorar o suporte do cuidado ${ }^{10}$.

A abordagem de observar a ocorrência dos sinais da doença como febre ou sintomas respiratórios (tosse seca, dor de garganta e falta de ar) que poderiam vir a ser sinais de alerta para a gravidade da doença ${ }^{3}$ e a suspensão de alguns procedimentos foram e são necessários para red-uzir os riscos da infecção pelo vírus ${ }^{11}$.

O controle da circulação de pessoas foi fundamental como medida de prevenção de novas contaminações, se fizeram e se fazem essenciais para evitar a disseminação desse vírus nos estabelecimentos de saúde $^{11}$. Assim como os novos EPIs que passaram a ser rotina dos profissionais $\mathrm{da}$ instituição, pois a proteção e segurança do trabalhador são barreiras importantíssimas à exposição do COVID-19 12 .

A inexistência de suporte adequado afetou diretamente o cuidado ofertado, gerando consequências imensuráveis à saúde dos trabalhadores e pacientes ${ }^{13}$. Do ponto de vista da saúde desses, foi vivido um dos piores cenários da história ${ }^{11}$. A pandemia levou à importante percepção de que a garantia do cuidado de pacientes e profissionais deve ser mantida independentemente da situação vivenciada ${ }^{14}$.

\section{CONCLUSÃO}

Diante do exposto fica evidente que a instituição passou por diversas modifica- ções e desafios frente ao enfrentamento da pandemia ocasionada pela doença do COVID-19. As mudanças na abordagem de trabalho refletem sobre o cenário caótico que encontrava-se a unidade de saúde no início das infecções pelo SARS-Cov-2. Além de adaptações estruturais, a instituição passou por uma gama de modificações assistenciais para melhor se adequar ao novo cenário vivenciado.

O planejamento de estratégias frente à nova realidade se mostrou primordial durante esse período. Sendo assim, evidente os desafios encontrados no percurso da assistência, mas contudo se fez essencial as novas adaptações. Dessa forma, é notório que o compromisso com o cuidado na exposição aos riscos à saúde dos pacientes e trabalhadores da linha de frente precisam seguir atualizações, afim de acompanhar as demandas necessárias a realidade que permeia o contexto atual da pandemia ocasionada pela doença do COVID-19.

\section{REFERÊNCIAS}

1. Aquino EML, Silveira IH, Pescarini JM, Aquino R, Sousa-Filho $J A$, Rocha AS, et al. Medidas de distanciamento social no controle da pandemia de COVID-19: potenciais impactos e desafios no Brasil. Ciênc.Saúde Coletiva. [Internet]. 2020. [acesso em 28 de janeiro de 2021]; 25 (1): 2423-2446.

2. World Health Organization. Oxygen sources and distribution for COVID-19 treatment centres: interim guidance. Geneva: World Health Organization. [Internet]. 2020. [cited 2021 jan 28].

3. Macedo TR, Possili L, Rocha D, Prado SS, Westphal PC. Regulação em saúde em tempos de Covid-19: um relato de experiência. Braz. J. Hea. [Internet]. 2020. [acesso em 26 de janeiro de 2021]. 3 (6): 19455- 19465.

4. Estevão A. COVID-19. Acta Radiológica Portuguesa. [Internet]. 2020. [acesso em 27 de janeiro de 2021]. 32(1):5-6.

5. Ministério da Saúde (Brasil). Secretaria de Vigilância em Saúde. Especial doença pelo coronavírus 2019. Boletim Epidemiológico 46 [Internet].2019. [acesso em 24 de janeiro de 2021].

6. Werneck GL, Carvalho MS. A pandemia de COVID-19 no Brasil: crônica de uma crise sanitária anunciada. Cad. de Saúde Pública. [Internet]. 2020. [acesso em 26 de janeiro de 2021]. 36 (5):00068820.

7. Medeiros EAS. A luta dos profissionais de saúde no enfrentamento da COVID-19. Acta Paul. Enferm. [Internet]. 2020. [acesso em 26 de janeiro de 2021]. 33: e-EDT20200003.

8. Rodrigues AKS, Oliveira Junior LB, Vieira BB, Camila R. Desafios da gestão de hospitais públicos brasileiros no cenário da pandemia COVID-19. HU Rev. [Internet]. 2020. [acesso em 25 de janeiro de 2021]. 46: 1-2.

9. Associação Nacional de Hospitais Particulares. Comunicação no ambiente hospitalar: como integrar o corpo clínico com a equipe assistencial. Anahp. [Internet]. 2016. [acesso em 26 de janeiro de 2021].

10. Organización Panamericana de la Salud. Recomendaciones para la reorganización y expansión de los servicios hospitalarios en respuesta a la COVID -19. OPS. [Internet].2020. [acceso 2021 jan 24].

11. Almeida IM. Proteção da saúde dos trabalhadores da saúde em tempos de COVID-19 e respostas à pandemia. Rev. Bras. Saúde Ocup. [Internet] 2020. [acesso em 26 de janeiro de 2021]. 45: e17.

12. Gallasch $\mathrm{CH}$, Cunha ML, Pereira LAS, Silva-Junior JS. Prevenção relacionada à exposição ocupacional do profissional de saúde no cenário de COVID-19. Ver. Enf.UERJ [Internet]. 2020. [acesso em 28 de janeiro de 2021]. 28 (1): e49596.

13. Chaves LA, Chaves GC, Vianna MNS, Oliveira MA. Desabastecimento de medicamentos na literatura científica da saúde: uma revisão narrativa. Physis:Rev.Saúde Coletiva. [Internet]. 2019. [ acesso em 27 de janeiro de 2021]. 29 (1):e290107.

14. Paula VRM, Paula GM, Linares FDC, Afonso TC.Enfrentando covid 19 em uma instituição hospitalar privada: relato de experiência. Braz. J. of Develop [Internet].2020. [acesso em 25 de janeiro de 2021]. 6 (11): 87727-87745. 